\title{
Influence of Various Temperatures on the Drying Time of 132 KV Transformer Insulations in Vapour Phase Drying Process
}

\author{
Mohd. Tareq Siddiqui, Jayant T. Pattiwar, Avinash P. Paranjape, Ashok J Keche
}

\begin{abstract}
Transformers are required to handle very high levels of voltage and hence proper insulation is very important in transformers. As of now, the most preferred form of insulation in transformers is cellulose based. The state of cellulose insulation materials like paper \& pressboards determines the life end of a transformer. Paper with 1.5\% moisture content ages 10 times faster than with only $0.3 \%$ moisture. For obvious reasons, it is very important that the moisture is removed from transformer insulation. Vacuum drying has been conventionally used in industries for insulation drying but, as of today the latest technology available is the vapour phase drying process. This paper evaluates the influence of temperatures at various locations on the drying time of the $132 \mathrm{kv}$ transformer insulations in vapour phase drying process.
\end{abstract}

Keywords: Temperature, drying time, moisture, transformer

\section{INTRODUCTION}

Transformer is required to withstand high voltages during the process of power transfer from primary to secondary. For this purpose, it is required to have adequate insulation. Due to long and positive experience, cellulose based insulation is widely used in transformer manufacturing \& it has a significant role in its life \& performance characteristics [1]. However, cellulose based insulation being a hygroscopic material, may contain 8 to $10 \%$ of moisture by weight at ambient temperature [2]. This moisture is injurious to the health of the transformers since it reduces the dielectric strength, raises the dielectric power factor, increases the risk of thermal breakdown of solid insulation, lowers the lowest hot-spot temperature range for possible bubble formation, accelerates thermal aging of paper insulation, and can be the root cause of a catastrophic failure [3]. It is therefore imperative to remove this moisture from the insulation. In the drying processes used for insulation drying, temperature attained in the insulation is one of the most important factors [4].

Revised Manuscript Received on February 05, 2020

* Correspondence Author

Mohd. Tareq Siddiqui*, Department of Mechanical Engineering, PES College of Engineering, Aurangabad, India. Email: mohd.tareq.siddiqui@gmail.com

Dr. Jayant T. Pattiwar, Department of Engineering \& Technology, Dr. BAM University, Aurangabad, India. Email: jayantpattiwar@gmail.com

Dr. Avinash P. Paranjape, Department of Electrical Engineering, Govt. Engineeirng College, Aurangabad, India. Email:

Dr. Ashok J. Keche Department of Mechanical Engineering, MIT College, Aurangabad, India. Email: ashok.keche@mit.asia

(C) The Authors. Published by Blue Eyes Intelligence Engineering and Sciences Publication (BEIESP). This is an open access article under the CC BY-NC-ND license (http://creativecommons.org/licenses/by-nc-nd/4.0/)
This paper presents a statistical analysis and evaluates the effects of temperatures at various locations viz., outer, middle \& innermost layers of insulation in transformer insulation drying using vapour phase drying process for two 132/27 KV, 45 MVA transformer coil with 2 ton insulation.

\section{VAPOUR PHASE DRYING OF 132KV (I)}

For the process of vapour phase drying, the 132/27 KV, 45 MVA transformer coil with 2 ton insulation was loaded into the vacuum chamber. The vacuum chamber has a provision of thermic fluid heating. Initially the chamber is evacuated for about 2 hours. The vacuum pressure of 19.17 mbar was observed at this point. Thereafter, the vacuum chamber is heated through thermic fluid for about 17 hours. It was observed at this point that the temperature of the outermost layer of insulation was $73^{\circ} \mathrm{C}$ while that of the middle layer was $67^{\circ} \mathrm{C}$ and that of the innermost layer was $62{ }^{\circ} \mathrm{C}$. Also, during this heating, the pressure in the vessel increased to 95mbar. The reason for this increase in pressure is the vapourisation of moisture form the outer layers of insulation. Then, the vacuum chamber is subjected to further pressure reduction for about 2 hours before kerosene vapours are introduced in the vacuum chamber. Kerosene vapours are introduced in the vacuum chamber for about 8 hours. As a result of injection of kerosene vapours, the temperature of the insulation increases such that the temperature of the outermost layer reaches up to $107^{\circ} \mathrm{C}$, while that of the middle layer is $103^{\circ} \mathrm{C}$ and that of the innermost layer is $99^{\circ} \mathrm{C}$. The final vacuum achieved at the end of the cycle was found to be 0.1 mbar. Also, the total amount of moisture removed was about 30 litres at the end of cycle.

The following tables illustrates the readings of temperatures and the moisture removal per hour during the drying cycle. In the table, $\mathrm{t} 1$ is the temperature of the outermost layer of the insulation, $\mathrm{t} 2$ is the temperature of the middle layer of insulation \& $\mathrm{t} 3$ is the temperature of the innermost layer of the insulation which is nearest to the core. It may be clearly seen from the table that the temperatures in the insulation are not the same throughout. Maximum temperature is observed on the outermost layer while, minimum temperature is observed on the innermost layer. The different temperatures at different locations in the insulation have different effect on the drying time of the insulation.

Table- I: Vacuum Chamber Readings (I)

\begin{tabular}{l|c|c|c|c|c|}
\hline $\begin{array}{c}\text { Time } \\
\text { in Hrs. }\end{array}$ & $\begin{array}{c}\text { Total } \\
\text { Water/ } \\
\text { Hr }\end{array}$ & $\mathbf{t 1}$ & $\mathbf{t 2}$ & $\mathbf{t 3}$ & $\begin{array}{c}\text { Vacuum } \\
\text { Level } \\
\text { (mbar) }\end{array}$ \\
\hline 0 & 0.27 & 33 & 33 & 33 & 975.42 \\
\hline 1 & 0.38 & 31 & 30 & 30 & 139.88 \\
\hline 2 & 0.45 & 37 & 31 & 29 & 19.17 \\
\hline 3 & 0.6 & 38 & 33 & 32 & 33.62 \\
\hline
\end{tabular}




\begin{tabular}{|c|c|c|c|c|c|}
\hline 4 & 0.71 & 40 & 35 & 34 & 35.97 \\
\hline 5 & 0.88 & 42 & 37 & 34 & 40.86 \\
\hline 6 & 0.97 & 46 & 40 & 35 & 39.56 \\
\hline 7 & 1.09 & 46 & 41 & 36 & 45.49 \\
\hline 8 & 1.28 & 48 & 43 & 36 & 50.42 \\
\hline 9 & 1.51 & 52 & 45 & 39 & 54.78 \\
\hline 10 & 1.73 & 54 & 47 & 42 & 62.56 \\
\hline 11 & 1.95 & 55 & 49 & 43 & 68.44 \\
\hline 12 & 2.15 & 58 & 51 & 45 & 76.77 \\
\hline 13 & 2.22 & 62 & 55 & 48 & 84.52 \\
\hline 14 & 2.54 & 65 & 59 & 53 & 90.78 \\
\hline 15 & 2.74 & 68 & 63 & 57 & 94.41 \\
\hline 16 & 2.89 & 71 & 65 & 60 & 94.36 \\
\hline 17 & 3.01 & 72 & 66 & 60 & 94.46 \\
\hline 18 & 3.12 & 72 & 67 & 62 & 95.49 \\
\hline 19 & 3.2 & 73 & 67 & 62 & 95.25 \\
\hline 20 & 3.08 & 77 & 70 & 65 & 71.76 \\
\hline 21 & 14.33 & 86 & 79 & 70 & 24.62 \\
\hline 22 & 25.17 & 95 & 85 & 77 & 12.97 \\
\hline 23 & 27.25 & 98 & 88 & 81 & 13.53 \\
\hline 24 & 27.25 & 101 & 90 & 83 & 2.3 \\
\hline 25 & 28.65 & 105 & 95 & 89 & 0.1 \\
\hline 26 & 29.46 & 103 & 98 & 91 & 0.1 \\
\hline 27 & 29.46 & 105 & 98 & 91 & 0.1 \\
\hline 28 & 29.46 & 107 & 101 & 97 & 0.1 \\
\hline 29 & 29.46 & 108 & 102 & 98 & 0.1 \\
\hline 30 & 29.46 & 107 & 103 & 99 & 0.1 \\
\hline
\end{tabular}

In the drying processes used for insulation drying, temperature attained in the insulation is one of the most important factors [4]. However, to establish temperature at which location in the insulation, will have the maximum influence on the drying time, Taguchi \& Regression analysis were performed using Minitab software between temperatures and drying time and temperatures \& rate of moisture removal.

\section{TAGUCHI ANALYSIS BETWEEN TEMPERATURES \& TIME (I)}

While maintaining the quality standards, it is always desirable to have lower drying times. Here quality pertains to the amount of moisture removed. Taguchi analysis between temperatures \& drying time was therefore performed by taking temperatures as input factors \& drying time as response variable. Signal to noise ratios were also evaluated for "Smaller the Better" (drying time). The values obtained from the analysis may summarized in tables $2 \& 3$.

Table- II: Response Table for Signal to Noise Ratios "Smaller Is Better" (I)

\begin{tabular}{|c|c|c|c|}
\hline Level & t1 & t2 & t3 \\
\hline 1 & 0 & 0 & -6.0206 \\
\hline 2 & & -6.0206 & 0 \\
\hline 3 & -6.0206 & -9.5424 & -9.5424 \\
\hline 4 & -9.5424 & -12.0412 & \\
\hline 5 & -12.0412 & -13.9794 & -13.0103 \\
\hline 6 & -13.9794 & -15.563 & -15.563 \\
\hline 7 & -16.2325 & -16.902 & -17.4819 \\
\hline 8 & -18.0618 & -18.0618 & -19.0849 \\
\hline 9 & -19.0849 & -19.0849 & -20 \\
\hline 10 & -20 & -20 & -20.8279 \\
\hline 11 & -20.8279 & -20.8279 & -21.5836 \\
\hline 12 & -21.5836 & -21.5836 & -22.2789 \\
\hline 13 & -22.2789 & -22.2789 & -22.9226 \\
\hline 14 & -22.9226 & -22.9226 & -23.5218 \\
\hline 15 & -23.5218 & -23.5218 & -24.3457 \\
\hline 16 & -24.0824 & -24.0824 & -25.3403 \\
\hline 17 & -24.8572 & -24.609 & -26.0206 \\
\hline 18 & -25.5751 & -25.3403 & -26.4444 \\
\hline 19 & -26.0206 & -26.0206 & -26.8485 \\
\hline 20 & -26.4444 & -26.4444 & -27.2346 \\
\hline 21 & -26.8485 & -26.8485 & -27.6042 \\
\hline 22 & -27.2346 & -27.2346 & -27.9588 \\
\hline 23 & -27.6042 & -27.6042 & -28.4634 \\
\hline 24 & -28.2995 & -27.9588 & -28.9432 \\
\hline 25 & -28.293 & -28.4634 & -29.248 \\
\hline 26 & -29.2428 & -28.9432 & -29.5424 \\
\hline 27 & -29.248 & -29.248 & \\
\hline 28 & & -29.5424 & \\
\hline Delta & 29.248 & 29.5424 & 29.5424 \\
\hline
\end{tabular}

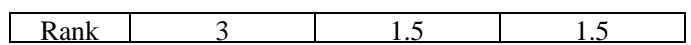

Table- III: Response Table for Means (I)

\begin{tabular}{|c|c|c|c|}
\hline Level & t1 & t2 & t3 \\
\hline 1 & 1 & 1 & 2 \\
\hline 2 & 0 & 2 & 1 \\
\hline 3 & 2 & 1.5 & 3 \\
\hline 4 & 3 & 4 & 0 \\
\hline 5 & 4 & 5 & 4.5 \\
\hline 6 & 5 & 6 & 6 \\
\hline 7 & 6.5 & 7 & 7.5 \\
\hline 8 & 8 & 8 & 9 \\
\hline 9 & 9 & 9 & 10 \\
\hline 10 & 10 & 10 & 11 \\
\hline 11 & 11 & 11 & 12 \\
\hline 12 & 12 & 12 & 13 \\
\hline 13 & 13 & 13 & 14 \\
\hline 14 & 14 & 14 & 15 \\
\hline 15 & 15 & 15 & 16.5 \\
\hline 16 & 16 & 16 & 18.5 \\
\hline 17 & 17.5 & 17 & 20 \\
\hline 18 & 19 & 18.5 & 21 \\
\hline 19 & 20 & 20 & 22 \\
\hline 20 & 21 & 21 & 23 \\
\hline 21 & 22 & 22 & 24 \\
\hline 22 & 23 & 23 & 25 \\
\hline 23 & 24 & 24 & 26.5 \\
\hline 24 & 26 & 25 & 28 \\
\hline 25 & 26 & 26.5 & 29 \\
\hline 26 & 29 & 28 & 30 \\
\hline 27 & 29 & 29 & \\
\hline 28 & & 30 & \\
\hline Delta & 29 & 29 & 30 \\
\hline Rank & 2.5 & 2.5 & 1 \\
\hline
\end{tabular}

The graphs generated are as follows.

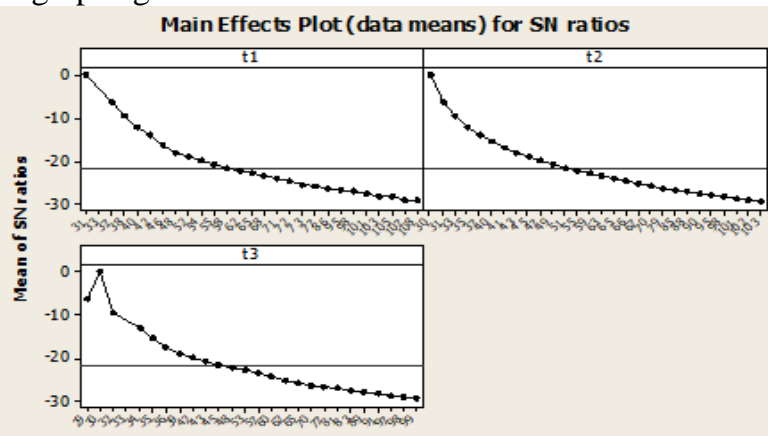

Signal-tonoise: Smaller is better

Main Effects Plot (data means) for Means

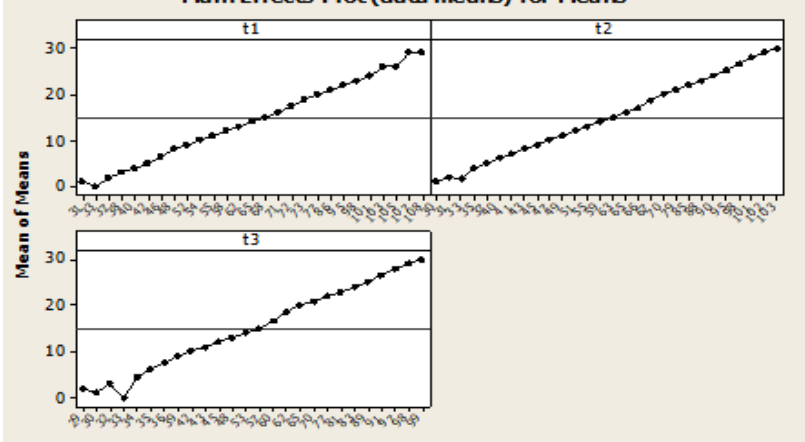

Fig. 1. Temp. Vs Time, Taguchi Graphs (I)

\section{REGRESSION ANALYSIS BETWEEN TEMPERATURES \& TIME. (I)}

To further asses which temperature has the maximum influence on the drying time, regression analysis was performed. Drying time was taken as the response \& temperatures was taken as the predictor. The regression equation obtained is as follows.

Published By:

Blue Eyes Intelligence Engineering 3073\& Sciences Publication 
Time in Hrs. $=-8.85-0.009 t 1+0.682 t 2-0.321 t 3$

(1)

Regression in tabular form can be illustrated as follows.

Table- IV: Regression Analysis of Temperatures Vs Time (I)

\begin{tabular}{|c|c|c|c|c|}
\hline Predictor & Coef & $\begin{array}{c}\text { SE } \\
\text { Coef }\end{array}$ & T & P \\
\hline Constant & -8.8506 & 0.7524 & -11.76 & 0 \\
\hline $\mathrm{t} 1$ & -0.0089 & 0.1459 & -0.06 & 0.952 \\
\hline $\mathrm{t} 2$ & 0.6818 & 0.2627 & 2.6 & 0.015 \\
\hline $\mathrm{t} 3$ & -0.3213 & 0.1498 & -2.15 & 0.041 \\
\hline
\end{tabular}

The regression graphs are as follows. Residual Plots for Time in Hrs.
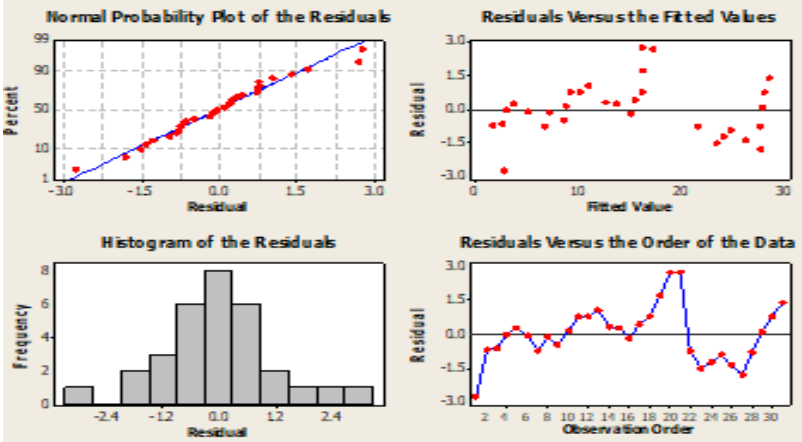

Fig. 2. Temp. Vs Time, Regression Graphs (I)

\section{TAGUCHI ANALYSIS BETWEEN TEMPERATURES \& MOISTURE REMOVAL RATE. (I)}

By taking temperatures as input factors \& moisture removal rate as response variable Taguchi analysis between temperatures \& moisture removal rate was performed to further determine the effect of different temperatures on the moisture removal rate and by extension on the drying time. Signal to noise ratios were also evaluated for "Larger the Better" (moisture removal rate). The values obtained from the analysis may summarized in tables $5 \& 6$.

Table- V: Response Table for Signal to Noise Ratios "Larger Is Better" (I)

\begin{tabular}{|c|c|c|c|}
\hline \\
\hline Level & t1 & t2 & t3 \\
\hline 1 & -8.4043 & -8.4043 & -6.9357 \\
\hline 2 & -11.3727 & -6.9357 & -8.4043 \\
\hline 3 & -6.9357 & -7.9048 & -4.437 \\
\hline 4 & -4.437 & -2.9748 & -11.3727 \\
\hline 5 & -2.9748 & -1.1103 & -2.0426 \\
\hline 6 & -1.1103 & -0.2646 & -0.2646 \\
\hline 7 & 0.242 & 0.7485 & 1.4464 \\
\hline 8 & 2.1442 & 2.1442 & 3.5795 \\
\hline 9 & 3.5795 & 3.5795 & 4.7609 \\
\hline 10 & 4.7609 & 4.7609 & 5.8007 \\
\hline 11 & 5.8007 & 5.8007 & 6.6488 \\
\hline 12 & 6.6488 & 6.6488 & 6.9271 \\
\hline 13 & 6.9271 & 6.9271 & 8.0967 \\
\hline 14 & 8.0967 & 8.0967 & 8.755 \\
\hline 15 & 8.755 & 8.755 & 9.3946 \\
\hline 16 & 9.218 & 9.218 & 9.993 \\
\hline 17 & 9.7272 & 9.5713 & 9.771 \\
\hline 18 & 10.103 & 9.993 & 23.1249 \\
\hline 19 & 9.771 & 9.771 & 28.0177 \\
\hline 20 & 23.1249 & 23.1249 & 28.7073 \\
\hline 21 & 28.0177 & 28.0177 & 28.7073 \\
\hline 22 & 28.7073 & 28.7073 & 29.1425 \\
\hline 23 & 28.7073 & 28.7073 & 29.3847 \\
\hline 24 & 29.3847 & 29.1425 & 29.3847 \\
\hline 25 & 29.2636 & 29.3847 & 29.3847 \\
\hline 26 & 29.3847 & 29.3847 & 29.3847 \\
\hline 27 & 29.3847 & 29.3847 & \\
\hline 28 & & 29.3847 & \\
\hline Delta & 40.7574 & 37.789 & 40.7574 \\
\hline Rank & 1.5 & 3 & 1.5 \\
\hline
\end{tabular}

Table- VI: Response Table for Means (I)

\begin{tabular}{|c|c|c|c|}
\hline Level & $\mathbf{t 1}$ & $\mathbf{t} \mathbf{2}$ & $\mathbf{t 3}$ \\
\hline 1 & 0.38 & 0.38 & 0.45 \\
\hline 2 & 0.27 & 0.45 & 0.38 \\
\hline 3 & 0.45 & 0.435 & 0.6 \\
\hline 4 & 0.6 & 0.71 & 0.27 \\
\hline 5 & 0.71 & 0.88 & 0.795 \\
\hline 6 & 0.88 & 0.97 & 0.97 \\
\hline 7 & 1.03 & 1.09 & 1.185 \\
\hline 8 & 1.28 & 1.28 & 1.51 \\
\hline 9 & 1.51 & 1.51 & 1.73 \\
\hline 10 & 1.73 & 1.73 & 1.95 \\
\hline 11 & 1.95 & 1.95 & 2.15 \\
\hline 12 & 2.15 & 2.15 & 2.22 \\
\hline 13 & 2.22 & 2.22 & 2.54 \\
\hline 14 & 2.54 & 2.54 & 2.74 \\
\hline 15 & 2.74 & 2.74 & 2.95 \\
\hline 16 & 2.89 & 2.89 & 3.16 \\
\hline 17 & 3.065 & 3.01 & 3.08 \\
\hline 18 & 3.2 & 3.16 & 14.33 \\
\hline 19 & 3.08 & 3.08 & 25.17 \\
\hline 20 & 14.33 & 14.33 & 27.25 \\
\hline 21 & 25.17 & 25.17 & 27.25 \\
\hline 22 & 27.25 & 27.25 & 28.65 \\
\hline 23 & 27.25 & 27.25 & 29.46 \\
\hline 24 & 29.46 & 28.65 & 29.46 \\
\hline 25 & 29.055 & 29.46 & 29.46 \\
\hline 26 & 29.46 & 29.46 & 29.46 \\
\hline 27 & 29.46 & 29.46 & \\
\hline 28 & & 29.46 & \\
\hline Delta & 29.19 & 29.08 & 29.19 \\
\hline & 1.5 & 3 & 1.5 \\
\hline
\end{tabular}

The graphs generated are as follows.
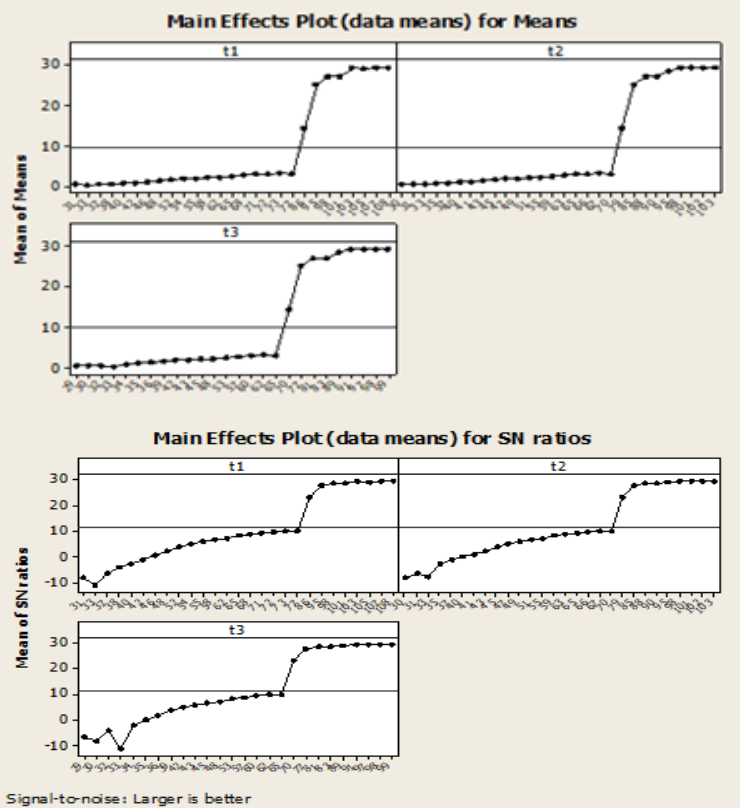

Fig. 3. Temp. Vs Moisture Removal Rate, Taguchi Graphs (I)

\section{REGRESSION ANALYSIS BETWEEN TEMPERATURES \& MOISTURE REMOVAL RATE. (I)}

Regression analysis was performed with moisture removal rate as the response \& temperatures as the predictor. The following regression equation was obtained.

Total Water $=-19.5+0.926 t 1-1.86 t 2+1.42 t 3$ (2)

Published By:

Plue Eyes Intelligence Engineering

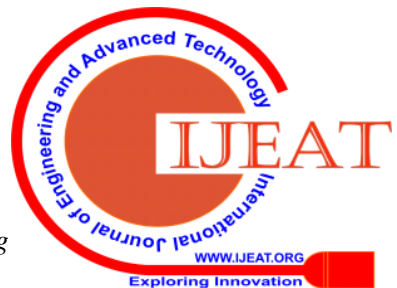


The following tabular form of regression was also obtained.

Table- VII: Regression Analysis of Temperatures Vs Moisture Removal Rate (I)

\begin{tabular}{|c|c|c|c|c|}
\hline Predictor & Coef & SE & T & P \\
\hline Constant & -19.514 & 2.78 & -7.02 & 0 \\
\hline t1 & 0.9257 & 0.5391 & 1.72 & 0.097 \\
\hline t2 & -1.8583 & 0.9707 & -1.91 & 0.066 \\
\hline t3 & 1.4192 & 0.5534 & 2.56 & 0.016 \\
\hline
\end{tabular}

The graphs generated are as follows.

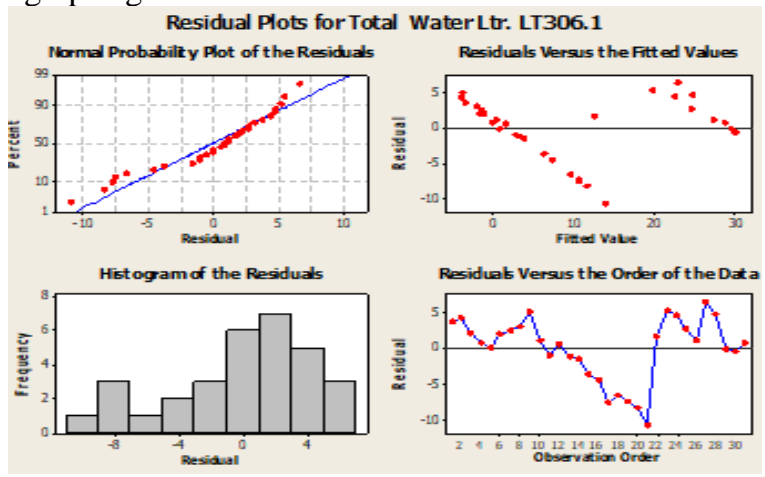

Fig 4.Temp. Vs Moisture Removal Rate, Regression Graphs. (I)

\section{VAPOUR PHASE DRYING OF 132KV (II)}

For the process of vapour phase drying, the 132/27 KV, 45 MVA transformer coil with 2 ton insulation was loaded into the vacuum chamber. Initially the chamber is evacuated for about 3 hours. The vacuum pressure of 20.81 mbar was observed at this point. Thereafter, the vacuum chamber is heated through thermic fluid for about 10 hours. It was observed at this point that the temperature of the outermost layer of insulation was $64^{\circ} \mathrm{C}$ while that of the middle layer was $60^{\circ} \mathrm{C}$ and that of the innermost layer was $54{ }^{\circ} \mathrm{C}$. Also, during this heating, the pressure in the vessel increased to 56.8 mbar. The reason for this increase in pressure is the vapourisation of moisture form the outer layers of insulation. Then, the vacuum chamber is subjected to further pressure reduction for about 3 hours. Next, a second heating cycle is taken for 2 hours during which the temperature of the outermost layer reaches $90{ }^{\circ} \mathrm{C}$ while that of the middle layer reaches $84^{\circ} \mathrm{C}$ and that of the innermost layer reaches $77^{\circ} \mathrm{C}$. the pressure observed at this point is $61.15 \mathrm{mbar}$. Kerosene vapours are introduced in the vacuum chamber for about 10 hours. As a result of injection of kerosene vapours, the temperature of the insulation increases such that the temperature of the outermost layer reaches up to $108^{\circ} \mathrm{c}$, while that of the middle layer is $102^{\circ} \mathrm{C}$ and that of the innermost layer is $101^{\circ} \mathrm{C}$. The final vacuum achieved at the end of the cycle was found to be 0.1 mbar. Also, the total amount of moisture removed was about 24 litres at the end of cycle. The following tables illustrates the readings of temperatures and the moisture removal per hour during the drying cycle.

Table-VIII: Vacuum Chamber Readings (II)

\begin{tabular}{|c|c|c|c|c|c|}
\hline $\begin{array}{c}\text { Time } \\
\text { in Hrs. }\end{array}$ & $\begin{array}{c}\text { Total } \\
\text { Water/ } \\
\text { Hr }\end{array}$ & t1 & t2 & t3 & $\begin{array}{c}\text { Vacuum } \\
\text { Level } \\
\text { (mbar) }\end{array}$ \\
\hline 0 & 0.38 & 37 & 37 & 37 & 971.06 \\
\hline 1 & 0.47 & 37 & 37 & 37 & 971.1 \\
\hline 2 & 0.69 & 37 & 36 & 36 & 96.71 \\
\hline 3 & 0.88 & 43 & 42 & 40 & 20.81 \\
\hline 4 & 1.08 & 44 & 43 & 43 & 29.4 \\
\hline 5 & 1.15 & 44 & 44 & 43 & 35.77 \\
\hline 6 & 1.21 & 45 & 44 & 43 & 38.99 \\
\hline
\end{tabular}

\begin{tabular}{|c|c|c|c|c|c|}
7 & 1.38 & 54 & 50 & 47 & 41.87 \\
\hline 8 & 1.44 & 60 & 55 & 50 & 47.73 \\
\hline 9 & 1.59 & 61 & 56 & 52 & 50.93 \\
\hline 10 & 1.63 & 61 & 55 & 53 & 52.3 \\
\hline 11 & 1.69 & 62 & 56 & 52 & 53.41 \\
\hline 12 & 1.72 & 64 & 60 & 54 & 56.8 \\
\hline 13 & 1.74 & 68 & 62 & 57 & 55.22 \\
\hline 14 & 2.65 & 75 & 69 & 63 & 52.16 \\
\hline 15 & 4.04 & 84 & 75 & 70 & 39.16 \\
\hline 16 & 4.05 & 85 & 77 & 72 & 53.77 \\
\hline 17 & 4.05 & 90 & 84 & 77 & 61.15 \\
\hline 18 & 5.14 & 94 & 90 & 84 & 38.69 \\
\hline 19 & 8.5 & 99 & 95 & 88 & 27.26 \\
\hline 20 & 13.53 & 100 & 96 & 92 & 10.63 \\
\hline 21 & 15.36 & 103 & 98 & 93 & 11.65 \\
\hline 22 & 18.67 & 105 & 99 & 95 & 0.16 \\
\hline 23 & 20.58 & 107 & 100 & 95 & 0.1 \\
\hline 24 & 23.49 & 107 & 100 & 96 & 0.1 \\
\hline 25 & 23.55 & 106 & 103 & 99 & 0.1 \\
\hline 26 & 23.57 & 106 & 102 & 100 & 0.1 \\
\hline 27 & 23.62 & 108 & 102 & 101 & 0.1 \\
\hline
\end{tabular}

\section{TAGUCHI ANALYSIS BETWEEN TEMPERATURES \& TIME. (II)}

Taguchi analysis between temperatures \& drying time was performed by taking temperatures as input factors \& drying time as response variable. Signal to noise ratios were also evaluated for "Smaller the Better" (drying time). The values obtained from the analysis may summarized in tables 9 \& 10.

Table- IX: Response Table for Signal to Noise Ratios "Smaller Is Better" (II)

\begin{tabular}{|c|c|c|c|}
\hline Level & $\mathbf{t 1}$ & $\mathbf{t} \mathbf{}$ & $\mathbf{t} 3$ \\
\hline 1 & -1.505 & -6.021 & -6.021 \\
\hline 2 & -9.542 & 3.01 & 3.01 \\
\hline 3 & -13.01 & -9.542 & -9.542 \\
\hline 4 & -15.563 & -12.041 & -13.861 \\
\hline 5 & -16.902 & -14.771 & -16.902 \\
\hline 6 & -18.062 & -16.902 & -18.062 \\
\hline 7 & -19.542 & -19.031 & -19.956 \\
\hline 8 & -20.828 & -19.956 & -20 \\
\hline 9 & -21.584 & -21.584 & -21.584 \\
\hline 10 & -22.279 & -22.279 & -22.279 \\
\hline 11 & -22.923 & -22.923 & -22.923 \\
\hline 12 & -23.522 & -23.522 & -23.522 \\
\hline 13 & -24.082 & -24.082 & -24.082 \\
\hline 14 & -24.609 & -24.609 & -24.609 \\
\hline 15 & -25.105 & -25.105 & -25.105 \\
\hline 16 & -25.575 & -25.575 & -25.575 \\
\hline 17 & -26.021 & -26.021 & -26.021 \\
\hline 18 & -26.444 & -26.444 & -26.444 \\
\hline 19 & -26.848 & -26.848 & -27.042 \\
\hline 20 & -28.129 & -27.419 & -27.604 \\
\hline 21 & -27.822 & -28.299 & -28.293 \\
\hline 22 & & -28.293 & -28.299 \\
\hline Delta & 26.624 & 31.31 & 31.31 \\
\hline Rank & 3 & 1.5 & 1.5 \\
\hline & & & \\
\hline
\end{tabular}


Table- X: Response Table for Means (II)

\begin{tabular}{|c|c|c|c|}
\hline Level & t1 & t2 & t3 \\
\hline 1 & 1.25 & 2 & 2 \\
\hline 2 & 3 & 0.5 & 0.5 \\
\hline 3 & 4.5 & 3 & 3 \\
\hline 4 & 6 & 4 & 5 \\
\hline 5 & 7 & 5.5 & 7 \\
\hline 6 & 8 & 7 & 8 \\
\hline 7 & 9.5 & 9 & 10 \\
\hline 8 & 11 & 10 & 10 \\
\hline 9 & 12 & 12 & 12 \\
\hline 10 & 13 & 13 & 13 \\
\hline 11 & 14 & 14 & 14 \\
\hline 12 & 15 & 15 & 15 \\
\hline 13 & 16 & 16 & 16 \\
\hline 14 & 17 & 17 & 17 \\
\hline 15 & 18 & 18 & 18 \\
\hline 16 & 19 & 19 & 19 \\
\hline 17 & 20 & 20 & 20 \\
\hline 18 & 21 & 21 & 21 \\
\hline 19 & 22 & 22 & 22.5 \\
\hline 20 & 25.5 & 23.5 & 24 \\
\hline 21 & 24.6667 & 26 & 26 \\
\hline 22 & & 26 & 26 \\
\hline Delta & 24.25 & 25.5 & 25.5 \\
\hline Rank & 3 & 1.5 & 1.5 \\
\hline
\end{tabular}

The graphs generated are as follows.

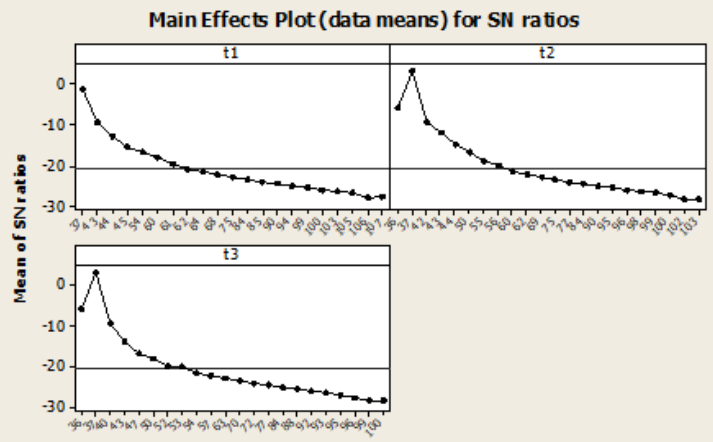

Signal-tonoise: Smaller is better

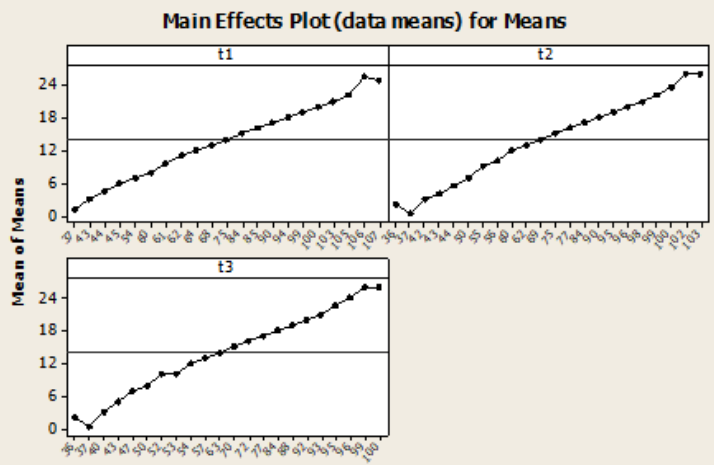

Fig. 5. Temp. Vs Time, Taguchi Graphs (II)

\section{REGRESSION ANALYSIS BETWEEN TEMPERATURES \& TIME. (II)}

To asses which temperature has the maximum influence on the drying time, regression analysis was performed. Drying time was taken as the response \& temperatures was taken as the predictor. The regression equation obtained is as follows.

Time in hrs $=-9.83+0.343 t 1-0.263 t 2+0.245 t 3$ (3)

Regression in tabular form can be illustrated as follows.

Table- XI: Regression Analysis of Temperatures Vs Time (II)

\begin{tabular}{|c|c|c|c|c|}
\hline Predictor & Coef & SE Coef & T & P \\
\hline Constant & -9.8316 & 0.8624 & -11.4 & 0 \\
\hline $\mathrm{t} 1$ & 0.3426 & 0.1678 & 2.04 & 0.052 \\
\hline
\end{tabular}

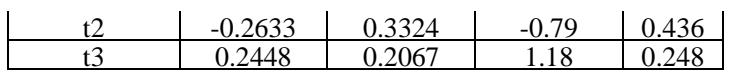

The regression graphs are as follows. Residual Plots for Time in hrs

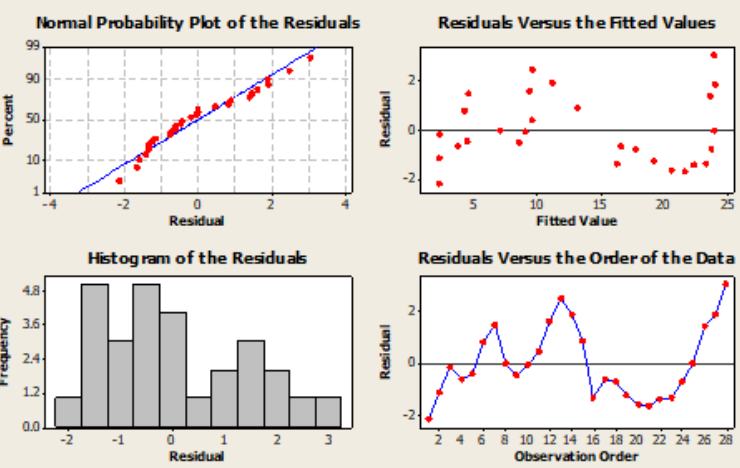

Fig. 6. Temp. Vs Time, Regression Graphs (II)

\section{TAGUCHI ANALYSIS BETWEEN TEMPERATURES \& MOISTURE REMOVAL RATE. (II)}

By taking temperatures as input factors \& moisture removal rate as response variable Taguchi analysis between temperatures \& moisture removal rate was performed to determine the effect of different temperatures on the moisture removal rate and by extension on the drying time. Signal to noise ratios were also evaluated for "Larger the Better" (moisture removal rate). The values obtained from the analysis may summarized in tables $12 \& 13$.

Table- XII: Response Table for Signal to Noise Ratios "Larger Is Better" (II)

\begin{tabular}{|c|c|c|c|}
\hline Level & t1 & t2 & t3 \\
\hline 1 & -5.4008 & -3.223 & -3.223 \\
\hline 2 & -1.1103 & -7.5786 & -7.5786 \\
\hline 3 & 0.9412 & -1.1103 & -1.1103 \\
\hline 4 & 1.6557 & 0.6685 & 1.1794 \\
\hline 5 & 2.7976 & 1.4348 & 2.7976 \\
\hline 6 & 3.1672 & 2.7976 & 3.1672 \\
\hline 7 & 4.1358 & 3.7055 & 4.2928 \\
\hline 8 & 4.5577 & 4.2928 & 4.2438 \\
\hline 9 & 4.7106 & 4.7106 & 4.7106 \\
\hline 10 & 4.811 & 4.811 & 4.811 \\
\hline 11 & 8.4649 & 8.4649 & 8.4649 \\
\hline 12 & 12.1276 & 12.1276 & 12.1276 \\
\hline 13 & 12.1491 & 12.1491 & 12.1491 \\
\hline 14 & 12.1491 & 12.1491 & 12.1491 \\
\hline 15 & 14.2193 & 14.2193 & 14.2193 \\
\hline 16 & 18.5884 & 18.5884 & 18.5884 \\
\hline 17 & 22.626 & 22.626 & 22.626 \\
\hline 18 & 23.7278 & 23.7278 & 23.7278 \\
\hline 19 & 25.4229 & 25.4229 & 25.8459 \\
\hline 20 & 27.4435 & 26.8433 & 27.4177 \\
\hline 21 & 27.0507 & 27.4472 & 27.4527 \\
\hline 22 & & 27.4472 & 27.4527 \\
\hline Delta & 32.8443 & 35.0313 & 35.0313 \\
\hline Rank & 3 & 1.5 & 1.5 \\
\hline
\end{tabular}


Table- XIII: Response Table for Means (II)

\begin{tabular}{|c|c|c|c|}
\hline Level & $\mathbf{t 1}$ & $\mathbf{t} \mathbf{2}$ & $\mathbf{t} 3$ \\
\hline 1 & 0.5575 & 0.69 & 0.69 \\
\hline 2 & 0.88 & 0.425 & 0.425 \\
\hline 3 & 1.115 & 0.88 & 0.88 \\
\hline 4 & 1.21 & 1.08 & 1.1467 \\
\hline 5 & 1.38 & 1.18 & 1.38 \\
\hline 6 & 1.44 & 1.38 & 1.44 \\
\hline 7 & 1.61 & 1.535 & 1.64 \\
\hline 8 & 1.69 & 1.64 & 1.63 \\
\hline 9 & 1.72 & 1.72 & 1.72 \\
\hline 10 & 1.74 & 1.74 & 1.74 \\
\hline 11 & 2.65 & 2.65 & 2.65 \\
\hline 12 & 4.04 & 4.04 & 4.04 \\
\hline 13 & 4.05 & 4.05 & 4.05 \\
\hline 14 & 4.05 & 4.05 & 4.05 \\
\hline 15 & 5.14 & 5.14 & 5.14 \\
\hline 16 & 8.5 & 8.5 & 8.5 \\
\hline 17 & 13.53 & 13.53 & 13.53 \\
\hline 18 & 15.36 & 15.36 & 15.36 \\
\hline 19 & 18.67 & 18.67 & 19.625 \\
\hline 20 & 23.56 & 22.035 & 23.49 \\
\hline 21 & 22.5633 & 23.57 & 23.585 \\
\hline 22 & & 23.57 & 23.585 \\
\hline Delta & 23.0025 & 23.16 & 23.16 \\
\hline Rank & 3 & 1.5 & 1.5 \\
\hline & & &
\end{tabular}

The graphs generated are as follows.

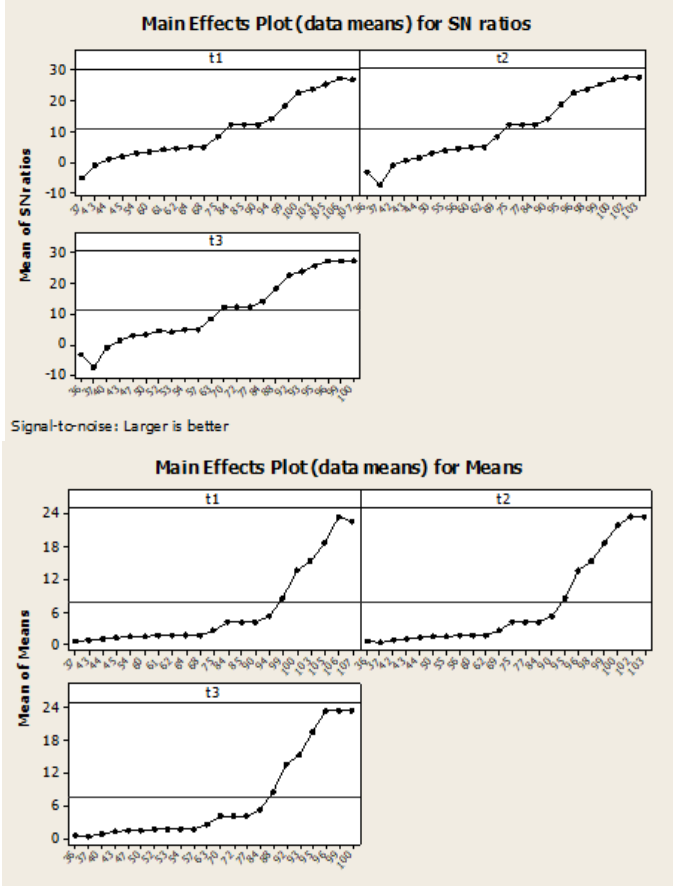

Fig. 7. Temp. Vs Moisture Removal Rate, Taguchi Graphs (II)

\section{REGRESSION ANALYSIS BETWEEN TEMPERATURES \& MOISTURE REMOVAL RATE. (II)}

Regression analysis was performed with moisture removal rate as the response \& temperatures as the predictor. The following regression equation was obtained.

Total Water $=-14.8+0.000 t 1-1.64 t 2+2.07 t 3$ (4)

The following tabular form of regression was also obtained.

Table- XIV: Regression Analysis of Temperatures Vs Moisture Removal Rate (I)

\begin{tabular}{|c|c|c|c|c|}
\hline Predictor & Coef & SE Coef & T & P \\
\hline Constant & -14.791 & 1.576 & -9.39 & 0 \\
\hline $\mathrm{t} 1$ & 0.0004 & 0.3066 & 0 & 0.999 \\
\hline $\mathrm{t} 2$ & -1.6444 & 0.6073 & -2.71 & 0.012 \\
\hline
\end{tabular}

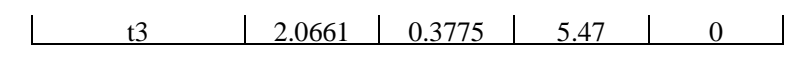

The graphs generated are as follows.

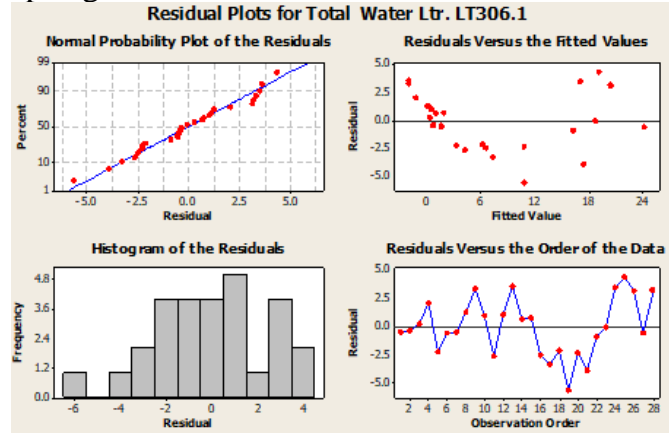

Fig. 8. Temp. Vs Moisture Removal Rate, Regression Graphs (II)

\section{RESULTS \& DISCUSSIONS}

Since the delta value and rank in Taguchi analysis (I) between temperatures and drying time is the highest for the innermost layer temperature as can be seen in tables $2 \& 3$, it is clearly evident that, of all the temperatures, the innermost layer temperature of insulation has maximum influence on the drying time.

As can be clearly seen in table 4 for regression analysis (I) between temperatures and drying time that, $\mathrm{P}$ value for temperature of the innermost layer of insulation is minimum after the $\mathrm{P}$ value for temperature of the middle layer. It can therefore be concluded that the temperature of the innermost layer is one of the most influential temperature for drying time.

As can be clearly seen in tables 5 \& 6 for Taguchi analysis (I) between temperatures and moisture removal rate that the delta value \& rank of the innermost layer temperature i.e t3 is highest, it can therefore be concluded that, of all the temperatures, the temperature of the innermost layer of insulation will have maximum influence on the moisture removal rate as the temperature of the outermost layer cannot be exceeded after a certain limit.

It is clearly evident from table 7 for regression analysis (I) between temperatures and moisture removal rate that, $\mathrm{P}$ value for temperature of the innermost layer of insulation $t 3$ is minimum \& hence it is the most influential temperature on the moisture removal rate.

The delta value and rank in tables 9 \& 10 for Taguchi analysis (II) between temperatures and drying time is the highest for the innermost layer temperature i.e $\mathrm{t} 3$. Therefore, it is clearly evident that, of all the temperatures, the innermost layer temperature of insulation has maximum influence on the drying time.

As can be clearly seen in table 11 for regression analysis (II) between temperatures and drying time that, $\mathrm{P}$ value for temperature of the innermost layer of insulation is minimum. It can therefore be concluded from regression analysis that the temperature of the innermost layer is the most influential temperature for drying time.

As can be clearly seen in tables 12 \& 13 for Taguchi analysis (II) between temperatures and moisture removal that the delta value \& rank of the innermost layer temperature i.e t3 is highest, it can therefore be concluded that, of all the temperatures, the temperature of the 
innermost layer of insulation will have maximum influence on the moisture removal rate.

In table 14 it can be seen that the $\mathrm{P}$ value in regression analysis (II) between temperatures and moisture removal rate is minimum for temperature of the innermost layer of insulation $\mathrm{t} 3$ \& hence it is the most influential temperature on the moisture removal rate.

\section{CONCLUSION}

Form the results of the Taguchi and Regression analysis obtained for vapour phase drying, it is clearly seen that, the temperature of the innermost layer of the insulation which is nearest to the transformer core is the most decisive temperature in reducing the overall drying time and increasing the moisture removal rate. The innermost layer temperature is predominant in positively influencing to reduce the drying time and increasing the moisture removal rate. It can therefore be concluded that if the temperature of the innermost layer of insulation is increased, the overall drying time may be reduced as, this increase in temperature will serve to increase the moisture removal rate.

\section{ACKNOWLEDGMENT}

The authors are immensely thankful to Chaitanya Electromagnets, MIDC, Waluj, Aurangabad for allowing to study and analyze the process transformer insulation drying process through vapour phase drying.

\section{REFERENCES}

1. Pierre Boss, Günter Rindlisbacher. "Practical experience with the drying of power transformers in the field, applying the $\mathrm{LFH}$ technology." CIGRE Session 2004. Paris, France: CIGRE, 2004. 1-8.

2. Anand Bhanpurkar, Ajay Bangar, Rajan Sharma, H.P.Tripathi. "Comparative analysis of moisture removing processes from transformer which are used to increase its efficiency." Global Journal of Researches in Engineering (Global Journals Inc. (USA)) 12, no. 5 (2012): 6-10.

3. "Water in Transformers" Rich Simonelli, Territory General Manager - Southeast SPX Transformer Solutions, Inc. Office 919300-1522, Email Rich.Simonelli@spx.com

4. Diego F. García, Belén García, Juan Carlos Burgos. "Analysis of the influence of low-frequency heating on transformer drying - Part 1: Theoretical analysis." International Journal Electrical Power and Energy Systems (Elsevier) 38, no. 1 (June 2012): 84-89.

5. M D Wilkinson \& P Dyer of Bowden Bros Ltd. UK "Continuous Moisture Management: Extending Transformer Service Life"

6. Stephanie Raetzke, Maik Koch \& Martin Anglhuber "Monitoring the Drying Process of Power Transformers Using Dielectric Response Analysis"

7. Stefan Zabeschek \& HelmutStrzala of Heidrich Vacuum Systems, Germany "Drying Of High Voltage Transformers In The Field With A Mobile Vapor Phase Drying Equipment"

\section{AUTHORS PROFILE}

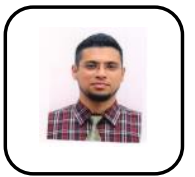

Mohd. Tareq Siddiqui is a research scholar in Dr.Babasaheb Ambedkar Marathwada University, Aurangabad pursuing his research in vapour phase drying of transformer insulation.

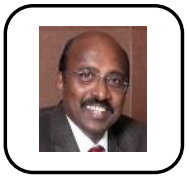

Dr. Jayant T. Pattiwar is a research guide in the faculty of science \& technology in Dr.Babasaheb Ambedkar Marathwada University, Aurangabad. His area of specialization is design. He has a vast experience in industry as well as academia.

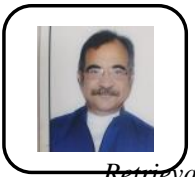

Dr. Avinash P. Paranjape is a professor emeritus in Govt. College of Engineering Aurangabad. He is a chartered engineer as well as a consultant for many industries in and around Aurangabad.

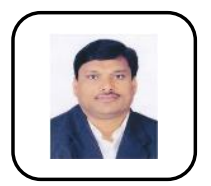

Dr. Ashok J. Keche is Head of the Department of Mechanical Engineering in MIT Aurangabad. His area of specialization is Machine Design and Manufacturing Engineering 